\title{
Acute Peripheral Arterial Thrombosis in COVID-19 Patients on Therapeutic Anticoagulation
}

\author{
Abhyuday Kumar ${ }^{1} \odot$, Neeraj Kumar ${ }^{2}$, Shettru K Arun $^{3} \odot$, Subhajit Ghosh ${ }^{4}$, Arunima Pattanayak $^{5} \odot$
}

\begin{abstract}
Coronavirus disease-2019 (COVID-19) disease is often complicated by venous thrombosis and embolism. However, arterial thrombosis in a patient already on therapeutic anticoagulation is a rare occurrence. In one patient, we found thrombosis of the radial artery leading to ischemia of the fingers and lateral part of the palm. In another patient, thrombosis was found in the anterior tibial artery, resulting in ischemia of the limb and amputation. Both these patients were on a therapeutic dose of low-molecular-weight heparin. In both cases, heparin infusion was started but with little benefit. It is suggested that intensified anticoagulation should be done in patients at high-risk venous thrombosis, and prompt interventional thrombectomy should be done in cases of failure of pharmacological anticoagulation.
\end{abstract}

Keywords: Artery, COVID-19, Intensive care, Low-molecular-weight heparin, Thrombosis.

Indian Journal of Critical Care Medicine (2021): 10.5005/jp-journals-10071-23793

\section{INTRODUCTION}

Coronavirus disease-2019 (COVID-19) disease is often complicated by venous thrombosis and embolism. In a recent meta-analysis, the incidence of venous thromboembolism was found to be $26 \%$; pulmonary embolism with or without deep vein thrombosis (DVT) occurred in $12 \%$ of patients and DVT alone in $14 \% .{ }^{1}$ However, data on arterial thrombosis in COVID-19 patients are limited.

Here, we describe two cases admitted to the intensive care unit (ICU) who developed acute peripheral arterial thrombosis despite on therapeutic anticoagulation leading to limb-threatening ischemia.

\section{Case Descriptions}

\section{Case 1}

A 66-year-old male COVID-19 patient with a history of diabetes and hypertension was admitted to the ICU with complaints of increased respiratory distress. He was put on invasive mechanical ventilation and was paralyzed because of severe acute respiratory distress syndrome requiring high oxygen concentration. He was started on antibiotics, hydroxychloroquine, dexamethasone, therapeutic dose of low-molecular-weight heparin (LMWH), and other supporting treatments. Both the left and right radial arteries were used for arterial blood gas analysis in due course of his stay in ICU. On day 3 of ICU admission, an arterial cannula was inserted in the right radial artery because of septic shock. On day 4, a red dusky discoloration was noticed on the tip of the left thumb, index, and little fingers. On Doppler ultrasonography (USG), the left ulnar artery was found to be patent, whereas the left radial artery was found to be thrombosed. The patient was started on an infusion of unfractionated heparin and aspirin. Overnight the tip of the index and little fingers turned to be red dusky to blackish, while the whole of left thumb was dark red (Fig. 1). Digital subtraction angiography (DSA) and thrombectomy were not performed due to clinical deterioration of the patient, and the patient died on day 6 due to sepsis and respiratory failure.

\footnotetext{
${ }^{1,3-5}$ Department of Anaesthesiology, All India Institute of Medical Sciences, Patna, Bihar, India

${ }^{2}$ Department of Trauma and Emergency, All India Institute of Medical Sciences, Patna, Bihar, India
}

Corresponding Author: Abhyuday Kumar, Department of Anaesthesiology, All India Institute of Medical Sciences, Patna, Bihar, India, Phone: +91 9013512403, e-mail: drabhyu@gmail.com

How to cite this article: Kumar A, Kumar N, Arun SK, Ghosh S, Pattanayak A. Acute Peripheral Arterial Thrombosis in COVID-19 Patients on Therapeutic Anticoagulation. Indian J Crit Care Med 2021;25(4):461-462.

Source of support: Nil

Conflict of interest: None

\section{Case 2}

A 75-year-old male COVID-19 patient with a history of hypertension and chronic obstructive pulmonary disease was shifted to the ICU on the fourth day of admission to the hospital because of respiratory distress and was put on noninvasive ventilation. The patient was started on inj.remdesivir, dexamethasone, antibiotics, and therapeutic dose of $\mathrm{LMWH}$, along with mechanical compression devices and other supportive management. On day 14 , the patient started complaining of pain in the right lower limb with discoloration of the dorsum of the foot. Doppler USG was performed, which showed right lower limb microthrombi occluding branches of the anterior tibial artery. The patient was started on unfractionated heparin, aspirin, and ticagrelor. However, the color of the distal part of the foot along with fingers started turning reddish black in due course (Fig. 2). The patient underwent right lower limb thrombectomy by DSA with balloon angioplasty on the 20th day of hospitalization. However, there was complete ischemia of the distal part of the foot with loss of sensation. The patient was weaned off the ventilator after 15 days and discharged with a plan of amputation after a clear line of demarcation is developed. Below the ankle, amputation of the dead necrotic tissues was done after 1 month of the discharge. 


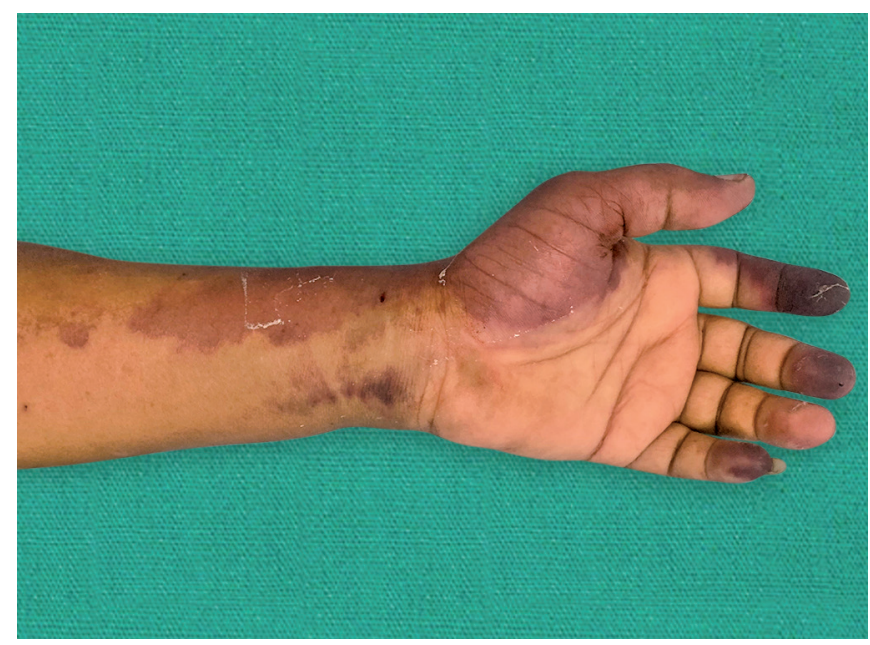

Fig. 1: Ischemic changes seen on the volar surface of palm and fingers
Table 1: Inflammatory markers on the day of clinical suspicion of thrombosis

\begin{tabular}{lll}
\hline Inflammatory markers & Case 1 & Case 2 \\
\hline Platelet count & 1.4 lac/cumm & 0.9 lac/cumm \\
C-reactive protein & $20.6 \mathrm{mg} / \mathrm{L}$ & $86 \mathrm{mg} / \mathrm{L}$ \\
D-dimer & $>20 \mu \mathrm{g} / \mathrm{mL}$ & $12.25 \mu \mathrm{g} / \mathrm{mL}$ \\
Lactate dehydrogenase & $2114 \mathrm{U} / \mathrm{L}$ & $515 \mathrm{U} / \mathrm{L}$ \\
Serum ferritin & $782 \mathrm{ng} / \mathrm{mL}$ & $362 \mathrm{ng} / \mathrm{mL}$ \\
Interleukin-6 & $110 \mathrm{pg} / \mathrm{mL}$ & $60 \mathrm{pg} / \mathrm{mL}$ \\
\hline
\end{tabular}

anticoagulation with LMWH. A formal investigation is warranted with a focus on arterial thrombotic complications to characterize the arterial consequences of COVID-19. Intensified anticoagulation should be done in patients with high venous thrombotic risk. Prompt interventional thrombectomy is also needed in cases of failure of pharmacological anticoagulation.

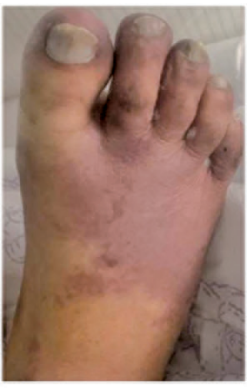

Day 14

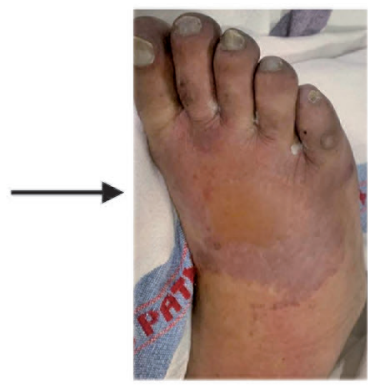

Day 16

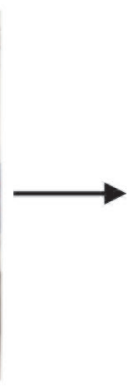

Fig. 2: Sequence of ischemic changes seen on dorsum and volar surfaces of the foot

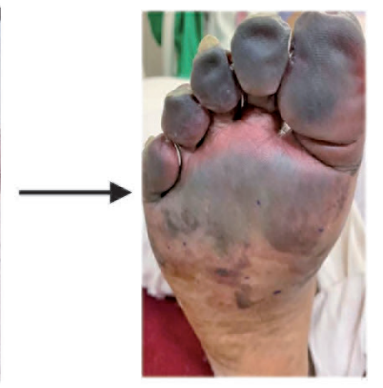

Day 20

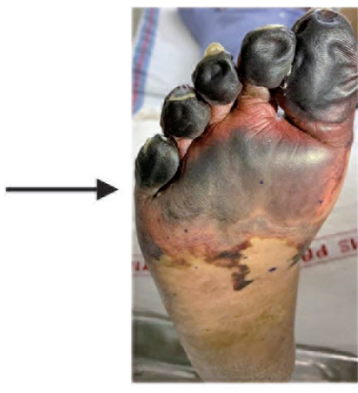

Day 25

\section{Discussion}

SARS-CoV-2 generates an imbalanced proinflammatory status (cytokine storm) with endothelial inflammation that can lead to venous or arterial thrombosis. ${ }^{2}$

SARS-CoV-2 directly attacks vascular endothelial cells and activates the coagulation cascade after causing endothelial injury. This results in cytokine storm activating the widespread coagulation factors while inhibiting the fibrinolysis causing extensive thrombosis similar to disseminated intravascular coagulation. Interleukin- 6 (IL-6) is a key factor in SARS-CoV-2-induced inflammatory storm. While IL-6 can stimulate the liver to synthesize fibrinogen and thrombopoietin, it also upregulates the expression of vascular endothelial growth factor to disrupt the stability of the vascular barrier and stimulate monocytes to express more tissue factors, thereby activating the extrinsic pathway of coagulation. ${ }^{3}$

D-dimer, platelet count, C-reactive protein, and erythrocyte sedimentation rate elevations have been found to be predictive of thrombotic complications. ${ }^{4}$ In our cases, D-dimer was found to be increased out of proportion in comparison with other inflammatory markers (Table 1). Prophylactic anticoagulation has been proved to be useful for venous thromboembolism; however, it is unclear whether prophylactic or full anticoagulation will be beneficial for arterial thrombosis caused by COVID-19. ${ }^{5}$ Our clinical scenario was unique in describing the development of acute limb ischemia due to arterial thrombosis despite being on full therapeutic

\section{ORCIDS}

Abhyuday Kumar @ https://orcid.org/0000-0002-9247-6713

Neeraj Kumar @ https://orcid.org/0000-0002-9161-7000

Shettru KArun @ https://orcid.org/0000-0002-9788-3350

Subhajit Ghosh @i https://orcid.org/0000-0001-9402-190X

Arunima Pattanayak @ https://orcid.org/0000-0001-6403-1517

\section{References}

1. Porfidia A, Valeriani E, Pola R, Porreca E, Rutjes AWS, Di Nisio M. Venous thromboembolism in patients with COVID-19: systematic review and meta-analysis. Thromb Res 2020;196:67-74. DOI: 10.1016/j. thromres.2020.08.020.

2. Gonzalez Cañas E, Gimenez Gaibar A, Rodriguez Lorenzo L, Castro Rios JG, Martinez Toiran A, Bella Cueto MR, et al. Acute peripheral arterial thrombosis in COVID-19. Role of endothelial inflammation. Br J Surg 2020;107:e444-e445. DOI: 10.1002/bjs.11904.

3. Zhou Y, Fu B, Zheng X, Wang D, Zhao C, Qi Y, et al. Aberrant pathogenic GM-CSF+T cells and inflammatory CD14+CD16+ monocytes in severe pulmonary syndrome patients of a new coronavirus. bioRxiv; 2020. DOI: 10.1101/2020.02.12.945576.

4. Al-Samkari H, Karp Leaf RS, Dzik WH, Carlson JCT, Fogerty AE, Waheed A, et al. COVID-19 and coagulation: bleeding and thrombotic manifestations of SARS-CoV-2 infection. Blood 2020;136(4):489-500. DOI: 10.1182/blood.2020006520.

5. Connors JM, Levy JH. COVID-19 and its implications for thrombosis and anticoagulation. Blood 2020;135:2033-2040. DOI: 10.1182/ blood.2020006000. 